\title{
Na ordering imprints a metallic kagomé lattice onto the Co planes of $\mathrm{Na}_{2 / 3} \mathrm{CoO}_{2}$
}

\author{
H. Alloul ${ }^{1}$ (a), I.R. Mukhamedshin ${ }^{1,2}$, T.A. Platova ${ }^{1,2}$ and A.V. Dooglav ${ }^{2}$ \\ '1 Laboratoie de Physique des Solides, UMR CNRS 8502, Univ. Paris-Sud, 91405 Orsay, France \\ 2 Physics Department, Kazan State University, 420008 Kazan, Russia
}

PACS 71.28.+d - Narrow-band systems; intermediate-valence solids

PACS 76.60.-k - Nuclear magnetic resonance and relaxation

PACS 71.27.+a - Strongly correlated electron systems; heavy fermions

\begin{abstract}
We report ${ }^{23} \mathrm{Na}$ and ${ }^{59} \mathrm{Co}$ nuclear magnetic (NMR) and quadrupolar resonance (NQR) studies for the $x=2 / 3$ phase of the lamellar oxide $\mathrm{Na}_{x} \mathrm{CoO}_{2}$, which allowed us to establish reliably the atomic order of the Na layers and their stacking between the $\mathrm{CoO}_{2}$ slabs. We evidence that the $\mathrm{Na}^{+}$order stabilizes filled non magnetic $\mathrm{Co}^{3+}$ ions on $25 \%$ of the cobalt sites arranged in a triangular sublattice. The transferred holes are delocalized on the $75 \%$ complementary cobalt sites which unexpectedly display a planar cobalt kagomé structure. These experimental results resolve a puzzling issue by precluding localized moments pictures for the magnetic properties. They establish that the quasi ferromagnetic properties result from a narrow band connecting a frustrated arrangement of atomic orbitals, and open the route to unravel through similar studies the electronic properties of the diverse ordered phases of sodium cobaltates.
\end{abstract}

Introduction. - Peculiar atomic structures such as chains, ladders or the graphene honeycomb often exhibit remarkable singular physical properties, as does the triangular cobalt network in Na cobaltates [1] which displays high thermopower [2] and superconductivity [3]. There, the control of carrier content of the $\mathrm{CoO}_{2}$ planes by varying $\mathrm{Na}$ concentration between the planes yields a totally counter-intuitive sequence of magnetic properties including anomalous paramagnetism [4], charge disproportionation [5], metallic antiferromagnetism [6,7].

An original aspect of these systems is that the Co atoms are stacked between two triangular oxygen layers with distorted $\mathrm{CoO}_{6}$ octahedrons, so that a large crystal field stabilizes the Co ions in a low spin state by lifting the degeneracy of the cobalt $3 d$ levels. In a $\mathrm{Co}^{3+}$ configuration the six lower energy levels $\left(t_{2 g}\right)$ are filled, with a total spin $S=0$, while $\mathrm{Co}^{4+}$ should only retain one hole in the $t_{2 g}$ multiplet, with $S=1 / 2$, so that original magnetic properties have been anticipated to result from these local spins associated with charge ordering [8]. This charge order intrinsic to the Co planes would depend of $x$ and would yield specific metallic and magnetic properties. For

\footnotetext{
(a) E-mail: alloul@lps.u-psud.fr
}

instance, for $x=2 / 3$, the $2 \mathrm{D}$ charge ordered state would be a honeycomb network of $\mathrm{Co}^{3+}$ ions intermixed with a triangular lattice of $\mathrm{Co}^{4+}$.

We formerly established experimentally by NMR [5] that such a charge order does not occur, but that the charges disproportionates between $\mathrm{Co}^{3+}$ and cobalt sites with an average formal valence of about $3.5^{+}$. The differentiation of three Na sites [11], in a phase with $x \sim 0.7$ (redefined as $x=0.67$ or H67 in ref. [9]) implied an associated atomic Na ordering with a unit cell larger than that of the honeycomb lattice, in view of the large number of cobalt sites detected by NMR [5]. This further differentiates the Na cobaltates from the cuprates for which copper has a uniform charge while dopants are usually disordered, which influences the physical properties [10]. Indeed the $\mathrm{Na}$ atomic orderings stabilized for specific $x$ values are found to play a role in determining the ground state metallic and magnetic properties [9]. But, while Na ordering has been evidenced by various experiments [11-13], the actual atomic order and its incidence on the local scale electronic properties is still unclear [9], except for two limiting cases, the $x=1$ filled band insulator and the $x=1 / 2$ ordered "chain-like" compound [14-16].

Let us point out that the cobalt plane physical prop- 
erties are influenced by the $\mathrm{Na}$ order in the two layers between which the $\mathrm{CoO}_{2}$ slab is sandwiched, so that any connection between structure and physical properties requires the knowledge of the $3 D$ structure. This is in principle possible using diffraction techniques and 3D order has indeed been seen by neutron scattering [13], X rays on single crystals [17] and even in our powder samples [9,18]. However, so far, materials complications linked with phase mixing, difficult accurate determination of $\mathrm{Na}$ content and existence of twins in the $\mathrm{Na}$ order have prevented the finalization of such studies.

It is well known that NMR is an ideal technique to link structural and electronic properties, as it allows to measure local magnetic properties and is also sensitive to structural properties. Indeed, nuclear spins with $I>1 / 2$ sense the local distribution of charges through the electrostatic interaction which couples the quadrupolar moment $e Q$ of the nuclear charge with the electric field gradient (EFG) at the nuclear site [19]. In view of the complicated NMR spectra [5], we demonstrate here that the structural and magnetic properties are better sorted out if one uses altogether NQR, that is a direct determination of the zero field splitting of the nuclear spin levels [19] due to the quadrupole interaction. We shall demonstrate below that this approach applied here to the $x=2 / 3$ phase allows us to evidence unambiguously that the $\mathrm{Na}$ ordering drives an in plane metallic electronic kagomé organization [20]. Efforts to synthesize such geometric structures at the atomic level have been successful recently on producing a remarkable $\mathrm{S}=1 / 2$ insulating spin liquid state $[21,22]$, but have not allowed so far to synthesize metallic states.

Differentiation of sites by NQR and NMR. We found that the simplest NMR spectra among the high sodium content phases [9] were observed for $x=0.67$. ${ }^{23} \mathrm{Na}$ NMR only exhibited three distinct Na sites on powder samples aligned within a polymer matrix by an applied field [11]. In such samples the crystallite grains were aligned along their $c$ direction while their $a, b$ directions are at random in the perpendicular plane (the alignment procedure was recalled in ref. [18] of our ref. [5]). The $\mathrm{Na}$ lines were not fully resolved, which could have been attributed to intrinsic sample disorder. In NQR, no magnetic field being applied, the spectra do not depend on the grain orientations. So the detection, in the same sample, of three well resolved narrow NQR lines (fig. 1h) with the expected quadrupolar frequencies $\nu_{Q}$, convinces us that the NMR resolution was limited by a small distribution of orientations of the crystallites.

For ${ }^{59} \mathrm{Co}$, we detected simple NQR spectra as well, with four well resolved sites (see fig. 10), grouped two by two, Co1a and Co1b with lower NQR frequencies than Co2a and Co2b. The overlap between the NMR spectra of these Co sites results in complicated NMR spectra as shown for instance in fig. 2. While the NMR differentiation techniques used in ref. [11] allowed us to separate there up to five ${ }^{59} \mathrm{Co}$ NMR signals, one of them was found to re-

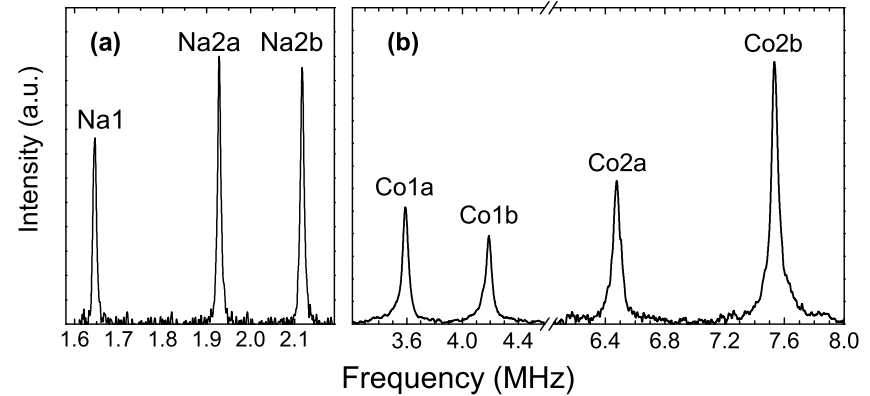

Fig. 1: NQR signals of the various ${ }^{23} \mathrm{Na}$ and ${ }^{59} \mathrm{Co}$ sites in $\mathrm{Na}_{2 / 3} \mathrm{CoO}_{2}$. (a) For ${ }^{23} \mathrm{Na}$, with $\mathrm{I}=3 / 2$, a single NQR line is expected per $\mathrm{Na}$ site, and indeed we detected three sites with narrow NQR linewidths. (b) For ${ }^{59} \mathrm{Co}$, with nuclear spin $I=$ $7 / 2$ we display here the four distinct lines corresponding to the higher frequency $(7 / 2 \rightarrow 5 / 2)$ transitions.

sult from a small fraction of polycrystalline grains of the sample which did not align in the field 1 .

The fractional occupancies of the various sites could be better fixed by comparing first the signal intensities of the sites with neighboring quadrupole frequencies. Similar ratios Co1a/Co1b=1.95(0.1) and Co2b/Co2a $=1.9(0.2)$ were obtained from NQR data of fig. 1a, after correcting for the spin-spin $T_{2}$ decay and for the $\nu^{2}$ frequency dependence of the signal intensity. The $\nu_{Q}$ values obtained by NQR allowed us to reduce as well the input parameters in the analysis of the ${ }^{59} \mathrm{Co}$ NMR spectra and to confirm this estimate of Co1a/Co1b $=1.85(0.2)$ and that Co2b has definitely a larger intensity than Co2a. To complete these comparisons one needed to determine the relative intensities of the Co1 and Co2 sites. The best accuracy has been obtained from a comparison of the total intensity of the Co1 sites signal to that of the full ${ }^{59} \mathrm{Co}$ NMR spectrum, which gave $\mathrm{Co} 1 /(\mathrm{Co} 1+\mathrm{Co} 2)=0.26(0.04)$. This allows to obtain the fractional occupancies given in table 1, which were quite helpful in the identification of the structure.

Lattice structure and site determination. - $\mathrm{Na}$ ordering patterns have been suggested both from diffraction experiments [13] and calculations restricted to electrostatic interactions between ions at fixed positions [13,24]. We found that the type of $\mathrm{Na}$ ordering proposed for $x \geq 0.75$, which consists in an ordering of droplets of Na2 tri-vacancies in which Na1 trimers are stabilized [13], cannot explain the present data (see fig. 3 for the usual convention taken for Na1 and Na2 sites). Such calculations are somewhat inconclusive on the possible stable structure for $x=2 / 3$. A recent quantum chemistry calculation

\footnotetext{
${ }^{1}$ Comparing different batches of samples, we noticed that the part of the spectrum corresponding to a large NMR shift for both directions of the field was sample dependent. It was erroneously assigned in the first experiments of ref. [11] to a site labelled Co3 with an "isotropic"shift. The analysis of the NMR shift data for the four Co sites will be detailed in a forthcoming publication (ref. [23]). The spectra shown in fig. 2 on a better oriented sample only retain the four site signals, in agreement with NQR.
} 
Table 1: EFG parameters $\nu_{Q}$ and $\eta$ for the different sites and relative intensities $I_{\text {exp }}$ deduced from NQR or NMR (see text). $I_{\text {exp }}$ can be compared to the intensities $I_{s t r}$ expected from the site occupancies $N_{s}$ of the structure depicted in fig. 3

\begin{tabular}{|c|c|c|c|c|c|}
\hline \multirow{2}{*}{ Site } & \multicolumn{3}{|c|}{ Experiment } & \multicolumn{2}{c|}{ Structure } \\
\cline { 2 - 6 } & $\nu_{Q}(\mathrm{MHz})$ & $\eta$ & $I_{\exp }(\%)$ & $N_{s}$ & $I_{\text {str }}(\%)$ \\
\hline Na1 & $1.645(5)$ & $<0.01$ & $30(5)$ & 2 & 25 \\
\hline Na2a & $1.74(1)$ & $0.84(2)$ & $33(5)$ & 3 & 37.5 \\
\hline Na2b & $1.87(1)$ & $0.89(2)$ & $37(5)$ & 3 & 37.5 \\
\hline Co1a & $1.193(2)$ & $<0.01$ & $17(3)$ & 2 & 16.7 \\
\hline Co1b & $1.392(2)$ & $<0.01$ & $9(2)$ & 1 & 8.3 \\
\hline Co2a & $2.187(3)$ & $0.36(1)$ & $25(3)$ & 3 & 25 \\
\hline Co2b & $2.542(3)$ & $0.36(1)$ & $49(4)$ & 6 & 50 \\
\hline
\end{tabular}

allowing the relaxation of the site positions [25] suggest for this $\mathrm{Na}$ content a relatively simple stable structure for $x=2 / 3$, with a $2 \mathrm{D}$ unit cell containing 12 cobalt atoms. It corresponds to $2 \mathrm{D}$ ordering of $\mathrm{Na} 2$ di-vacancies which stabilize a single Na1 site at their center in a position with axial symmetry. This unit cell which is shown on top of fig. 3a contains $8 \mathrm{Na}$ for $12 \mathrm{Co}$ atoms in the Co layer.

We shall see that we could fit perfectly NMR/NQR data by choosing the stacking pattern of this unit cell shown in fig. 3a, which is in good agreement with the "rules" suggested in ref. [25], i.e. minimize the number of Co sites with two Na1 above and below. The differentiation of sites intrinsic to this structure gives $4 \mathrm{Co}$ and $3 \mathrm{Na}$ sites, which are distinguished in fig. $3 \mathrm{~b}$ and fig. 3b. We find that six layers of $\mathrm{CoO}_{2}$ are required to complete the 3D unit cell shown in fig. 3r. This corresponds to a lattice parameter $3 c$, where $c$ is the usual unit cell length involving two $\mathrm{CoO}_{2}$ layers in the basic hexagonal structure, in agreement with the observation done on this phase by $\mathrm{X}$ rays [9]. Although this unit cell contains 72 cobalt, 144 oxygen and 48 sodium atoms, the local site differentiation of fig. $3 \mathrm{~b}, \mathrm{~b}$ is maintained in all the 3D crystal structure, with the in plane multiplicities $N_{s}$ of the 4 Co and $3 \mathrm{Na}$ sites given in table1. The agreement found there with the relative intensities of the corresponding NMR/NQR lines allows an unambiguous assignment of the different lines to the sites as given in fig. 3 $\mathrm{b}$. The Cola line which has the lower intensity is naturally assigned to the Co site of the 2D unit cell which has Na1 sites above and below, while Co1b corresponds to the two cobalt with one Na1 on one side and three $\mathrm{Na} 2$ on the other side (fig. 3b). The two other sites with multiplicity 3 and 6 are then naturally assigned to $\mathrm{Co} 2 \mathrm{a}$ and $\mathrm{Co} 2 \mathrm{~b}$, while the relative intensity of 0.26(0.04) found for the Co1 spectrum agrees perfectly with the expected 3:12 ratio for the structure of fig. 3a.

As for the ${ }^{23} \mathrm{Na}$ NMR data [11], the lower intensity line corresponds then to the Nal sites, while the two others with similar intensities can be indifferently assigned to $\mathrm{Na} 2 \mathrm{a}$ and Na2b. Let us point out that Chou et al. [26] reported, on a single crystal, a well resolved ${ }^{23} \mathrm{Na}$ spec-

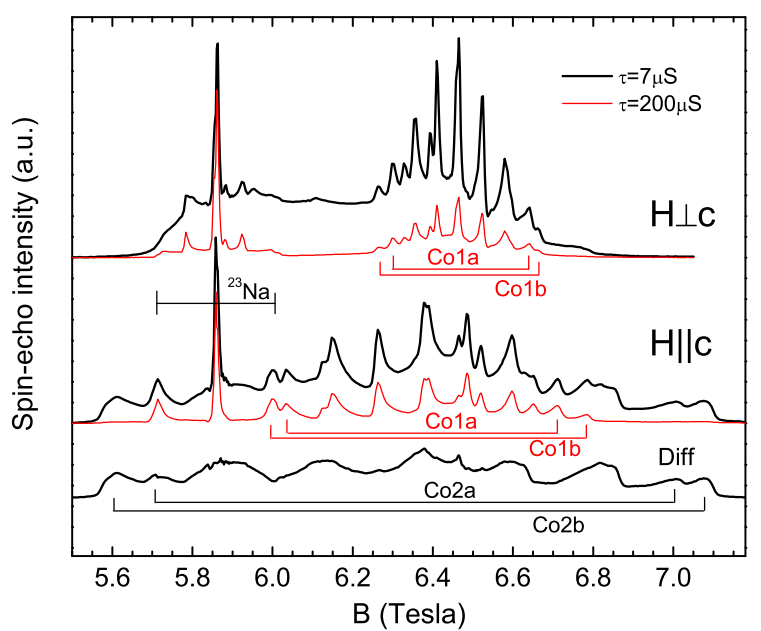

Fig. 2: Asymmetry of the ${ }^{59}$ Co NMR spectra. ${ }^{59}$ Co NMR spectra obtained by sweeping the applied field at fixed frequency and monitoring the NMR signal by the magnitude of the spin echo signal. The top spectra are taken with the external field applied parallel to the ab plane, while the lower ones are taken with $H \| c$. The black spectra taken with short delay between pulses $(\tau=7 \mu \mathrm{sec})$ give the overall NMR signal including all sites. The red spectra of the non magnetic Co1 sites, which have longer transverse spin-spin relaxation time, are separated out using a longer delay $(\tau=200 \mu \mathrm{sec})$ Scaling it to deduce it from the full spectrum (as in ref. [5]) allows to deduce the signal of the Co2 sites displayed as Diff. in the lower panel for $H \| c$. We have marked the positions of the $(5 / 2 \rightarrow 7 / 2)$ outer quadrupolar transitions for the $\mathrm{Co} 1$ and $\mathrm{Co} 2$ spectra for $H \| c$. For $H \perp c$ those are still well resolved for the Co1 with an extension reduced by a factor 2 (as marked). This is not the case for the Co2 signals for which $\eta \neq 0$.

trum taken at $20 \mathrm{~K}$ that we could ascribe without ambiguity to the same $\mathrm{H} 67$ phase, from the ${ }^{23} \mathrm{Na}$ NMR shifts and quadrupolar splitting values. They attempted to assign this three line spectrum to a phase with $x=0.71$, as obtained by the chemical analysis of their sample. However the relative intensities reported, $26.5 \%, 36.3 \%, 37.1 \%$, correspond extremely well within their $3 \%$ accuracy to the 2:3:3 ratio for the Na sites, which independently supports the validity of the structure proposed here.

Symmetry of the sites. - An important cross-test of the validity of this structure is the symmetry of the various sites, which could be probed through that of the EFG tensor. The NQR frequencies are indeed determined by the principal values of the EFG tensor, which is diagonal in a frame $(X, Y, Z)$ linked with the crystallographic structure $\left(\left|V_{Z Z}\right| \geq\left|V_{Y Y}\right| \geq\left|V_{X X}\right|\right)$. Laplace equation reduces those into two parameters, the quadrupolar frequency

$$
\nu_{Q}=3 e Q V_{Z Z} /[2 I(2 I-1)]
$$




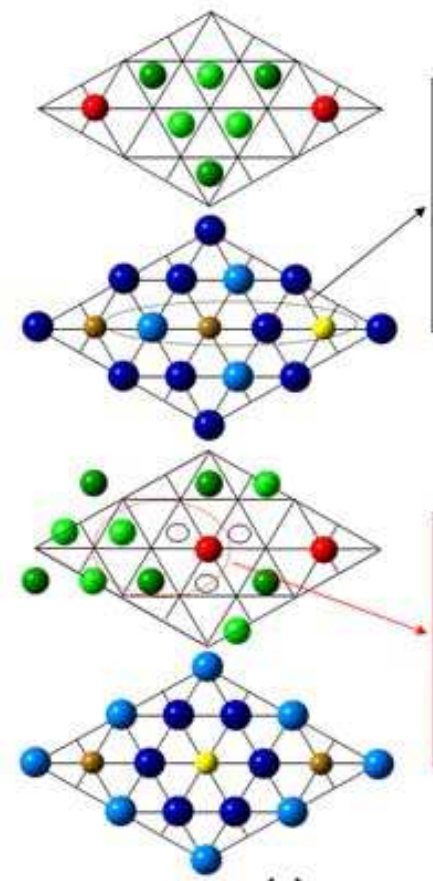

(a)

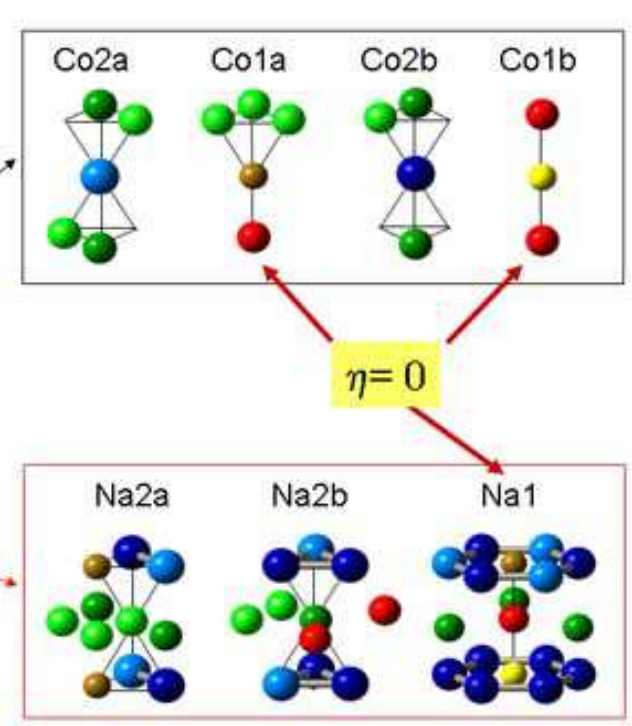

(b)

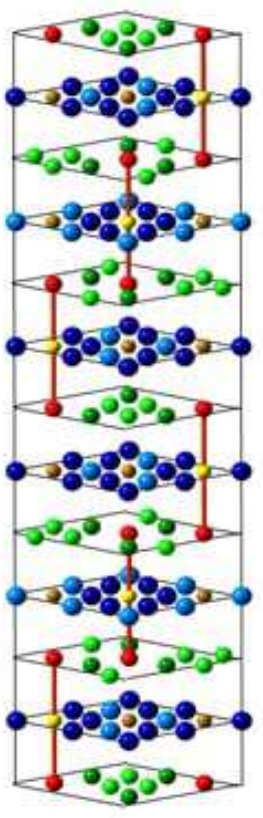

(c)

Fig. 3: Three dimensional structure of $\mathrm{Na}_{2} / 3 \mathrm{CoO}_{2}$. (a) The two dimensional Na unit cell proposed in ref. [25] is represented in the top layer with respect to the 12 initially indistinguishable Co atoms of the underlying triangular layer (the oxygen atoms layers above and below the cobalt layer are omitted). It contains two Na1 sites (red) sitting on top of Co and six Na2 sites, topping a triangle of $\mathrm{Co}$, with two distinct $\mathrm{Na}$ planar environments (light and dark green). The 3D stacking which fits our data involves a lower $\mathrm{Na}$ layer shifted by $-2 \boldsymbol{a}$. As the $\mathrm{Na} 2$ sites of the two layers should project at the centers of distinct $\mathrm{Co}$ triangles, $\boldsymbol{a}$ axis mirroring of the Na pattern is required. This planar Na structure can be considered as an ordered pattern of di-vacancies in the $\mathrm{Na} 2$ plane, which displace a third $\mathrm{Na} 2$ on an Na1 site in a three fold symmetric environment (one such di-vacancy is illustrated in the lower Na plane, where circles mark the three missing Na2 sites). (b) In the upper panel, the Na environments of the four Co sites differentiated in this structure are displayed (the smaller size of the orange and yellow balls is meant to distinguish Co sites with Na1 neighbors). In the lower panel, the $3 \mathrm{Na}$ sites are represented with their $\mathrm{Na}$ and Co environments deduced from the stacking of cobalt planes shown in (a). Three fold rotation symmetry is seen for Cola , Co1b and Na1 for which $\eta=0$. (c) The four layers of (a) are represented at the top and the 3D stacking of the following Na layers is pursued downwards by $-2 \boldsymbol{a}$ translations and mirroring, which shifts the Colb site by $-2 \boldsymbol{a}$ between Co layers (red bars). The unit cell is completed after six shifts. 
$\mathrm{Na}$ ordering imprints a metallic kagomé lattice onto the Co planes of $\mathrm{Na}_{2 / 3} \mathrm{CoO}_{2}$

and the asymmetry parameter

$$
\eta=\left(V_{X X}-V_{Y Y}\right) / V_{Z Z}
$$

which vanishes identically for sites with axial symmetry.

From fig. 3b it is easy to see that this is the case for the Na1 site as well as the Co1a and Co1b sites, for which $c$ is a threefold symmetry axis in the proposed structure. In NMR the weaker intensity Na site had been found [11] with $\eta=0.01(1)$, confirming the identification done with the Na1 site, while the Na2a and $\mathrm{Na} 2 \mathrm{~b}$ have large $\eta$ values in agreement with the non axial structure. Similarly, one can see in fig. 2 that the quadrupole splitting of the ${ }^{59} \mathrm{Co}$ NMR of the Co1 sites is reduced by a factor two in the $a b$ direction with respect to that in the $c$ direction. This is expected for sites with a threefold or fourfold symmetry axis for which $V_{X X}=V_{Y Y}=-V_{Z Z} / 2$, which corresponds to an axial symmetry for the EFG. In such a case the quadrupole splitting is identical for all field directions in the $a b$ plane, so that the NMR spectra are still well resolved for $H \perp c$, even for a powder distribution of the $a b$ orientations, as seen in fig. 2. On the contrary one can see that the $H \perp c$ spectra of the Co2 sites are less resolved and have a field extension comparable with that in the $H \| c$ direction, implying $\eta \neq 0$. A full study of the NMR and NQR spectra to be published elsewhere allowed us to deduce $\eta=0.36(1)$ for both $\mathrm{Co} 2$ sites.

So we do find that all the sites with axial symmetry of fig. 3b have been properly identified, which secures then the validity of the proposed structure. This confirms our $x=0.67(1)$ estimate for the Na content of this phase and establishes the validity of the relationship between $c$ axis parameter and $\mathrm{Na}$ content reported in $[9,18]$. We believe that other calibrations $[17,26]$ based on chemical analyses of samples overestimate the actual content of the majority phase from which $\mathrm{Na}$ tends to be expelled. This is particularly true for single crystal samples synthesized with a large excess of $\mathrm{Na}$, while for our single phase ceramic samples reacted in solid state, $\mathrm{Na}$ excess with respect to nominal content is unlikely.

Magnetic properties of the Co sites. - It has been shown for long that this particular phase has an anomalous Curie Weiss behavior extending down to the lowest temperatures [11] and a resistivity which only reaches a $T^{2}$ dependence below $2 \mathrm{~K}[27]$. The local magnetic parameters could be studied through NMR shift data [5] or spin lattice relaxation $T_{1}$ measurements which probe directly the dynamic susceptibilities. Here, to compare in detail the magnetic properties of the four cobalt sites we took advantage of the fact that the NQR lines are fully resolved spectrally in fig. 1] contrary to the NMR spectra of fig. 2 which largely overlap. This allowed us to probe independently the $T_{1}$ values of the four sites through monitoring the recovery of each NQR signal after saturation. The typical data taken at $4.2 \mathrm{~K}$ shown in fig. 4 immediately allow us to evidence that the Co2a and Co2b sites have identical $T_{1}$ values, which are about 30 times shorter
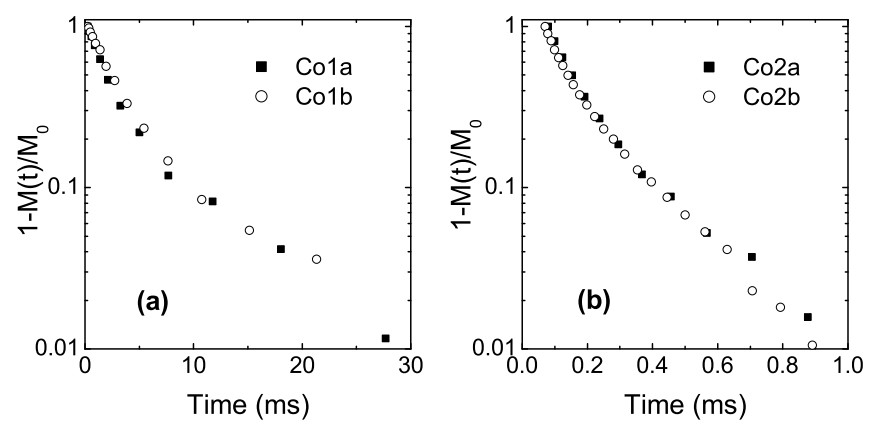

Fig. 4: Spin lattice relaxation of the Co sites taken at $4.2 \mathrm{~K}$. The magnetization recoveries of the ${ }^{59} \mathrm{Co}$ NQR signals are seen in (a) to be identical for the lower frequency signals of Cola and Co1b sites, and similarly in (b) for the higher frequency signals of $\mathrm{Co} 2 \mathrm{a}$ and $\mathrm{Co} 2 \mathrm{~b}$. The relaxation rate is about 30 times larger for the latter, which differentiates markedly the magnetic Co2 sites from the non magnetic Co1 sites.

than that of the non magnetic Cola and Co1b sites. For the latter the $T_{1}$ values are identical as well as the NMR shifts, which have an isotropic orbital component, which allowed us to evidence that they correspond to non magnetic $\mathrm{Co}^{3+}$ sites $[5,9]$. This illustrates that the magnetic properties are sustained by the Co2 sites which sense directly the on site magnetism that yields much larger spin lattice relaxation rates (and NMR shifts [5]). On the contrary, the $\mathrm{Co} 1$ and $\mathrm{Na}$ sense the magnetism of the $\mathrm{Co} 2$ sites only through transferred hyperfine couplings. For instance the fact that $\mathrm{Na} 2 \mathrm{a}$ and $\mathrm{Na} 2 \mathrm{~b}$ display smaller NMR shifts than Na1 [11] can be assigned to the larger number of magnetic Co2 near neighbours for the latter (fig. $3 \mathrm{~b}$ ).

More importantly the present results prove that Co2a and Co2b sites, although quite distinguished by their EFG values induced by the local structure, are quite identical on their electronic and magnetic properties. So the $\mathrm{Na}$ organization above and below the Co plane allows to pin $\mathrm{Co}^{3+}$ ionic states on the Co1 sites, by lowering their energy levels so that holes do only occur on the Co2 sites. For the understanding of the electronic properties one should then consider only two types of electronic sites (3 Co1 and $9 \mathrm{Co} 2$ ) in a single plane unit cell as shown in fig. 5. There one can notice that the Co2 sites are quite remarkably organized on a kagomé structure while the $\mathrm{Co}^{3+}$ sites form the complementary triangular lattice. The fractional occupancy 1:4 of the Co1 sites agrees well with the previous estimates of the $\mathrm{Co}^{3+}$ concentration, which allows us to establish that the partly filled Co2 sites bear then a hole concentration of $4 / 9$ per site, i.e. an effective charge $\mathrm{Co}^{3.44+}$, similar to that deduced before $[5,9]$, and far less than expected for a $\mathrm{Co}^{3+} / \mathrm{Co}^{4+}$ scenario.

Let us point out that a similar ordered charge disproportionation (OCD) has been found in the case of nickelates [28], but with opposite magnetic effects, i.e. local moments on one site out of three and charge delocalisation on the others. The experimental situation here is rather 
analogous to that found in the cubic $\mathrm{CsC}_{60}$ compounds where local singlets are favoured on some $\mathrm{C}_{60}$ balls while electrons delocalize on the remaining ones [29].

Discussion. - So far, most electrostatic calculations do give indications on possible $\mathrm{Na}$ orderings, but they do not introduce the electronic energies sufficiently well to give any insight on the electronic order associated. The calculation of ref. [25] which takes into account the electronic band energy (with a variant of the Local Density Approximation called GGA) yields an atomic structure in agreement with that proposed here. This underlines the importance of the electronic energy in pinning the $\mathrm{Na}$ order. Although the authors establish that the same $\mathrm{Na}$ structure is stable if a large on site $U$ is introduced in the GGA, nothing in the reported results helps so far to establish the stability of the OCD of fig. [5. The competition between Hund's energy and band energy which stabilizes local moments in the case of nickelates [28] might rather favour the filling of selected $\mathrm{Co}^{3+}$ sites in the present case. It would certainly be helpful to extend then the GGA calculations to check whether this approach is sufficient to explain a decrease of the ground sate energy by the OCD, or whether one has to resort to a more refined treatment of the electronic correlations. The derived band structure would then allow comparisons with the hyperfine and EFG parameters determined from our NMR data. Depending which bands $\left(a_{1 g}\right.$ and/or $\left.e_{g}^{\prime}\right)$ participate to the Fermi surface in the reduced Brillouin zone, might allow one to interpret the small pockets detected by transport experiments [30]. It might also be worth to attempt ARPES experiments [31] on ordered $\mathrm{Na}$ phases on the external layer of single crystals to detect their imprint on the Fermi surface, although this appears an experimental challenge.

On theoretical grounds, one would like as well to understand whether the Kagomé structure has any specific role in the physics of cobaltates, as had been underlined by Koshibae and Maekawa [32]. These authors anticipated that the directionality of the transfer integrals between Co sites in the triangular lattice favors electronic wave functions restricted to orbitals organized on a kagomé lattice. In a uniformly charged case four such interpenetrating lattices are degenerate, but the Na self organization might merely select here one of these kagomé lattices?

As for the magnetic properties of the cobaltates, the experimental evidence given here that metallicity and magnetism are combined in the correlated electron kagomé structure is at odds with most proposals concerning the electronic structure of cobaltates [8,33-35]. It has indeed most often been anticipated that $\mathrm{Na} 2$ vacancies pin localized spins which would dominate the Curie Weiss magnetic properties. In these approaches, close to the ionic $\mathrm{Co}^{3+} /$ $\mathrm{Co}^{4+}$ scenario, the metallic properties are associated with quasi non magnetic $\mathrm{Co}^{3+}$ sites assumed to retain $\mathrm{Na}$ configurations similar to that of $\mathrm{Na}_{1} \mathrm{CoO}_{2}$. On the contrary, our results establish that the Na1 sites are linked to non magnetic $\mathrm{Co}^{3+}$ on the $\mathrm{Co} 1$, and that metallicity occurs on

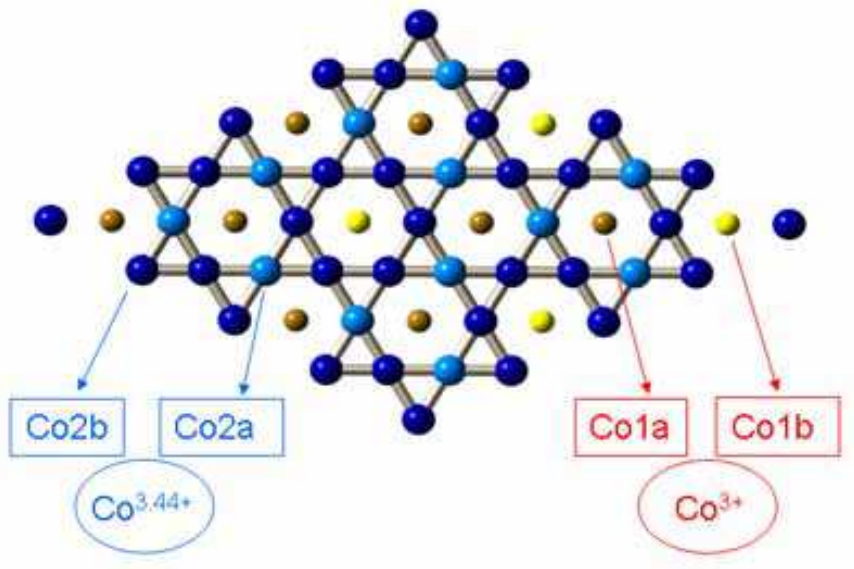

Fig. 5: Two dimensional charge distribution in the Co planes of $\mathrm{Na}_{2 / 3} \mathrm{CoO}_{2}$. The resulting 2D structure of the Co planes corresponds to a perfect Co2 kagomé lattice (fig. 35), once the minor difference between the electronic properties of Co2a and Co2b sites is neglected. The Co1 sites constitute the complementary triangular lattice.

the $\mathrm{Co} 2$ sites which, as viewed in fig. $3 \mathrm{~b}$, have a low Na coordinance ( 3 or 4 ) compared to the 6 fold one of $\mathrm{Na}_{1} \mathrm{CoO}_{2}$.

So the present results give a simple realistic structure on which approaches taking better into account the electronic correlations, such as DMFT [35] could be applied. It might even be used as a benchmark to develop and test the capabilities of electronic structure calculations of correlated electron systems. This could possibly permit to understand altogether why the dominant electronic correlations are ferromagnetic for $x \geq 2 / 3$ [9] and AF below [18]. Also with this well characterized 3D order one should understand whether specific inter-plane exchange paths could justify the absence for $x=2 / 3$ of the A type AF 3D order $[6,7]$ found for $x>0.75$. Conversely one might wonder whether this difference can be explained by an incidence of the 2D frustration inherent to the kagomé structure, establishing for instance an underlying spin liquid component as found in the $S=1 / 2$ kagomé lattice [22]? The present results not only raise these questions but open a path to solve them, as the experimental approach highlighted here, which combines the structural determination with its local impact on magnetism can be extended to the diverse cobaltate phases with distinct ground state properties.

Aknowledgments. - We acknowledge J. Bobroff, G. Collin, G. Lang, P. Mendels, M. Rozenberg and F. Rullier-Albenque for their constant interest, for helpful discussions and comments concerning the manuscript. We acknowledge financial support by the ANR (NT05-441913) in France. Expenses in Orsay for A. D. and I. M. have been supported by the "Triangle de la Physique". T. Platova has obtained a fellowship from the E.U. Marie Curie program "Emergentcondmatphys" for part of her $\mathrm{PhD}$ work performed in Orsay. 


\section{REFERENCES}

[1] Founassier, C., Matjeka G., Reau J.M., and HagenMuller P., Solid State Chem., 6 (1973) 532.

[2] Terasaki I., Sasago Y., and Uchinokura K., Phys. Rev. B, 56 (1997) R12685.

[3] TAKADA K. et al., Nature, 422 (2003) 53.

[4] Foo M.L. et al., Phys. Rev. Lett., 92 (2004) 247001.

[5] Mukhamedshin I. R., Alloul H., Collin G. and Blanchard N., Phys. Rev. Lett., 94 (2005) 247602.

[6] Bayrakci S.P. et al., Phys. Rev. B, 69 (2004) 100410(R).

[7] Helme, L.M., Boothroyd, A.T., Coldea, R. and Prabhakaran, D., Phys. Rev. B, 73 (2006) 054405.

[8] Baskaran, G., Phys. Rev. Lett., 91 (2003) 097003.

[9] Alloul, H., Mukhamedshin, I. R., Collin, G. and BlanChard, N., EPL, 82 (2008) 17002.

[10] F. Rullier-Albenque, H. Alloul, F. Balakirev and C. Proust, EPL, 81 (2008) 37008.

[11] Mukhamedshin I. R., Alloul H., Collin G. and Blanchard N., Phys. Rev. Lett., 93 (2004) 167601.

[12] Zandbergen H. W.et al., Phys. Rev. B, 70 (2004) 024101.

[13] Roger M. et al., Nature, 445 (2007) 631.

[14] Bobroff J. et al., Phys. Rev. Lett, 96 (2006) 107201.

[15] Yokoi M. et al., J. Phys. Soc. Japan, 74 (2005) 3046.

[16] Mendels P. et al., Phys. Rev. Lett., 94 (2005) 136403.

[17] Shu G.J. et al., Phys. Rev. B, 76 (2007) 184115.

[18] Lang G.et al., Phys. Rev. B, 78 (2008) 155116.

[19] Slichter C.P., "Principles of Magnetic Resonance" (Springer) 1978.

[20] Mekata M., Physics Today, 56 (2003) 12.

[21] Shores, M.P, Nytko, E.A., BArlett, B.M. and NoCERA, D.G., J. Am. Chem. Soc., 127 (2005) 13462.

[22] Mendels P. et al., Phys. Rev. Lett., 98 (2007) 077204.

[23] Mukhamedshin I.R. et al., unpublished, () .

[24] Zhang, P., Capaz, R.B., Cohen, M.L. and Louie, S.G., Phys. Rev. B, 71 (2005) 153102.

[25] Hinuma, Y., Meng, Y.S. and Ceder, G., Phys. Rev. B, 77 (2008) 224111.

[26] Chou, F.C. et al., arXiv:cond-mat/0709.0085, (2007) .

[27] Li S.Y. et al.,Phys. Rev.Lett., 93 (2004) 056401.

[28] Wawrzyńska, E. et al., Phys. Rev. Lett., 99 (2007) 157204.

[29] Brouet, V., Alloul, H., and Forro, L., Phys. Rev. B, 66 (2002) 155123.

[30] Balicas, L., Jo, Y.J., Shu, G.J., Chou, F.C. and Lee, P.A., Phys. Rev. Lett., 100 (2008) 126405.

[31] Yang, H.B. et al., Phys. Rev. Lett., 95 (2005) 146401.

[32] Koshibae, W. and Maekawa, S., Phys. Rev. Lett., 91 (2003) 257003.

[33] Bernhardt C. et al., Phys. Rev. Lett, 93 (2004) 167003.

[34] Chou F.C. et al., Phys. Rev. Lett., 101 (2008) 127404.

[35] Marianetti, C. A. and Kotliar, G., Phys. Rev. Lett., 98 (2007) 176405. 